\title{
Three-Dimensional Poisson Solver for a Charged Beam with Large Aspect Ratio in a Conducting Pipe
}

\author{
Ji Qiang \\ Lawrence Berkeley National Laboratory, Berkeley, CA 94720 \\ Robert L. Gluckstern \\ University of Maryland, College-Park, Maryland 20742
}

\begin{abstract}
In this paper, we present a three-dimensional Poisson equation solver for the electrostatic potential of a charged beam with large longitudinal to transverse aspect ratio in a straight and a bent conducting pipe with open-end boundary conditions. In this solver, we have used a Hermite-Gaussian series to represent the longitudinal spatial dependence of the charge density and the electric potential. Using the Hermite-Gaussian approximation, the original three-dimensional Poisson equation has been reduced into a group of coupled two-dimensional partial differential equations with the coupling strength proportional to the inverse square of the longitudinal-to-transverse aspect ratio. For a large aspect ratio, the coupling is weak. These two-dimensional partial differential equations can be solved independently using an iterative approach. The iterations converge quickly due to the large aspect ratio of the beam. For a transverse round conducting pipe, the two-dimensional Poisson equation is solved using a Bessel function approximation and a Fourier function approximation. The three-dimensional Poisson solver can have important applications in the study of the space-charge effects in the high intensity proton storge ring accelerator or induction linear accelerator for heavy ion fusion where the ratio of bunch length to the transverse size is large.
\end{abstract}

PACS numbers: 52.65.Rr; 52.75.Di

Keywords: Poisson solver, Hermite-Gaussian approximation, large aspect ratio, open boundary conditions 


\section{INTRODUCTION}

Solving the three-dimensional (3D) Poisson equation for the electrostatic potential of a charged long beam bunch in a conducting pipe has important applications in beam dynamics studies of modern accelerator physics. Recently, there has been increasing interest in utilizing high intensity beams for future accelerator applications, e.g, accelerator-driven spallation neutron source (SNS), and Fermilab booster accelerator upgrade. In these applications, the nonlinear space-charge forces from the charged particle interactions can cause particle loss, which results in the radioactivation of the accelerators. To minmize particle losses, selfconsistent particle-in-cell (PIC) models have been developed to simulate charged particle motion in the accelerators [1-3]. These models include both the space-charge forces from the beam and the forces from complex external fields. To calculate the space-charge forces, we must solve the Poisson equation for a given charge density distribution. A key issue in the PIC simulations is to solve the Poisson equation efficiently, at each time step, subject to the appropriate boundary conditions. In many applications, the computation of the electrostatic potential of a long bunch beam inside a conducting pipe with open ends is of particular importance.

In previous studies, a number of methods for solving Poisson's equation in a closed computational domain have been studied [4-8]. To use these methods for solving the Poisson equation in a conducing pipe with open end boundary conditions requires a large computation domain so that the potential vanishes at the ends of the domain. This is inefficient for beam dynamics studies, since only the potential inside the beam is needed. Furthermore, the choice of computational domain is not straightforward and usually requires solving Poisson's equation twice to ensure that the computational domain is large enough. An efficient method has been proposed in our previous study using a boundary matching procedure [9]. However, that method used a finite difference scheme for the longitudinal discretization. For a long bunch with very large longitudinal to transverse aspect ratio, e.g. $>100$, the use of a finite difference method in the longitudinal direction is computational efficient. For example, in the SNS accelerator, the beam has a longitudinal size of order of 100 meters while the transerse size of the beam is only a few centimeters. Putting a computational mesh on such a beam would require a large number of grid points longitudinally in order to get sufficient resolution. On the other hand, the spectral function approximation can have a much higher 
accuracy than the finite difference approximation. The open boundary conditions that the electric potential disappears at infinite distance leads to a natural choice of the HermiteGaussian function as a basis function. After approximating the longitudinal dependence of the electric potential and the charge density distribution using a Hermite-Gaussian series, we obtain a group of coupled two-dimensional (2D) partial differential equations (PDEs). Solving these coupled two-dimensional PDEs presents the same challenge as the original 3D Poisson equation. Fortunately, for a beam with large aspect ratio, the coupling strength of these two dimensioal PDEs is proportional to the inverse square of the aspect ratio. This suggests that for a beam with a large aspect ratio, the coupling between individual 2D PDEs for each longitudinal mode is weak. We have used an iterative approach and treated the coupling terms as source terms. This reduces the original 3D Poisson equation to a group of $2 \mathrm{D}$ Poisson equations. The iteration converges very quickly due to the weak coupling resulting from a large aspect ratio. The resulting two-dimensional Poisson equations are solved using a Bessel function and a Fourier function approximation in a transverse round conducting pipe. For a charged beam bunch in a bent conducting pipe, e.g. in storage ring accelerator, we have written the 3D Poisson equation in Frenet-Serret coordinates. The contribution from the curved structure has a multipler proportional to the ratio of pipe transverse size to the curvature radius. For most accelerators with curvature radius varying from 1 to 1000 meters, this ratio would be of order $10^{-2}$ to $10^{-5}$. Again this term can be treated as a perturbative source term in the above iterative approach and the iteration converges quickly.

The organization of this paper is as follows: The physical model and numerical methods are described in Section II. The numerical tests of the 3D Poisson solver are presented in Section III. The conclusions are drawn in Section IV.

\section{NUMERICAL METHODS}

We first discuss the solution of the 3D Poisson equation in a straight conducting pipe. For a pipe with circular cross section, we write the dimensionless Poisson equation in cylindric coordinates as:

$$
\frac{\partial^{2} \phi}{\partial r^{2}}+\frac{1}{r} \frac{\partial \phi}{\partial r}+\frac{1}{r^{2}} \frac{\partial^{2} \phi}{\partial \theta^{2}}+\frac{\partial^{2} \phi}{\partial z^{2}}=-\rho
$$


Here, $\phi$ denotes the dimensionless electrostatic potential, $\rho$ the dimensionless charge density function, $r$ and $z$ the dimensionless radial and longitudinal distance. The boundary conditions for the potential are:

$$
\begin{aligned}
\phi(r=1, \theta, z) & =0 \\
\phi(r, \theta+2 \pi, z) & =\phi(r, \theta, z) \\
\phi(r, \theta, z= \pm \infty) & =0
\end{aligned}
$$

Here, we have chosen the radial pipe radius $a$ as the length scale so that the radial boundary condition is set at $r=1$. The charge density $\rho$ and electrostatic potential $\phi$ can be approximated using a Hermite-Gaussian series along the $z$-axis so that the longitudinal boundary conditions in Eq. 4 are naturally satistifed.

$$
\begin{aligned}
\rho(r, \theta, z) & =\sum_{n=0}^{n=N} \rho_{n}(r, \theta) \mathcal{H}_{n}(z) \\
\phi(r, \theta, z) & =\sum_{n=0}^{n=N} \phi_{n}(r, \theta) \mathcal{H}_{n}(z)
\end{aligned}
$$

The scaled Hermite-Gaussian function $\mathcal{H}_{n}$ is defined as

$$
\mathcal{H}_{n}(z)=H_{n}\left(\frac{z}{A}\right) \exp \left(-\frac{1}{2} \frac{z^{2}}{A^{2}}\right)
$$

where $A$ is the dimensionless longitudinal scaling constant, which is the longitudinal to transverse aspect ratio, $A=\sigma_{z} / a$ with $\sigma_{z}$ the longitudinal beam rms size, $H_{n}$ is the $n t h$ order Hermite polynominal with properties: $H_{0}(z)=1, H_{1}(z)=2 z, \cdots, H_{n}(z)=2 z H_{n-1}-$ 2(n-1) $H_{n-2}$. The scaled Hermite-Gaussian function $\mathcal{H}$ has the properties:

$$
\begin{aligned}
\int_{-\infty}^{\infty} \mathcal{H}_{n}(z) \mathcal{H}_{m}(z) d z & =2^{n} n ! \sqrt{\pi} A \delta_{n m} \\
\frac{\partial^{2} \mathcal{H}_{n}}{\partial z^{2}} & =\frac{1}{4 A^{2}} \mathcal{H}_{n+2}+\frac{n(n-1)}{A^{2}} \mathcal{H}_{n-2}-\frac{2 n+1}{2 A^{2}} \mathcal{H}_{n}
\end{aligned}
$$

where $\delta_{m n}=1$ for $m=n$ and $\delta_{m n}=0$ for $m \neq n$. The expansion coefficients $\rho_{n}$ and $\phi_{n}$ can be obtained from

$$
\begin{aligned}
\rho_{n}(r, \theta) & =\frac{1}{2^{n} n ! \sqrt{\pi} A} \int_{-\infty}^{\infty} \rho(r, \theta, z) \mathcal{H}_{n}(z) d z \\
\phi_{n}(r, \theta) & =\frac{1}{2^{n} n ! \sqrt{\pi} A} \int_{-\infty}^{\infty} \phi(r, \theta, z) \mathcal{H}_{n}(z) d z
\end{aligned}
$$


Substituting the functions $\rho$ and $\phi$ into the 3D Poisson Eq. 1, and using the orthorgonality of the scaled Hermite-Gaussian functions and Eq. 9, the 3D Poisson equation is reduced to a group of coupled 2D PDEs with each equation satisfying

$$
\nabla_{\perp}^{2} \phi_{n}+\left(\frac{1}{4} \phi_{n-2}-\frac{1}{2}(2 n+1) \phi_{n}+(n+2)(n+1) \phi_{n+2}\right) \frac{1}{A^{2}}=-\rho_{n}
$$

where $\nabla_{\perp}^{2}$ is the transverse Laplace operator $\nabla_{\perp}^{2}=\frac{\partial^{2}}{\partial r^{2}}+\frac{1}{r} \frac{\partial}{\partial r}+\frac{1}{r^{2}} \frac{\partial^{2}}{\partial \theta^{2}}$. Eq. 12 is a group of coupled two-dimensional partial differential equations. Each longitudinal mode $n$ is coupled to the mode $n-2$ and $n+2$ with a coupling multiplier $\frac{1}{A^{2}}$. For a large longitudinal to transverse aspect ratio $A$, the coupling between different modes is weak. The contributions from the coupling modes in the second term of Eq. 12 can be treated as a perturbative source term using an iterative approach. The detailed procedure is as follows:

$$
\begin{aligned}
\nabla_{\perp}^{2} \phi_{n}^{1} & =-\rho_{n} \\
\nabla_{\perp}^{2} \phi_{n}^{2} & =-\rho_{n}-\left(\frac{1}{4} \phi_{n-2}^{1}-\frac{1}{2}(2 n+1) \phi_{n}^{1}+(n+2)(n+1) \phi_{n+2}^{1}\right) \frac{1}{A^{2}} \\
\nabla_{\perp}^{2} \phi_{n}^{3} & =-\rho_{n}-\left(\frac{1}{4} \phi_{n-2}^{2}-\frac{1}{2}(2 n+1) \phi_{n}^{2}+(n+2)(n+1) \phi_{n+2}^{2}\right) \frac{1}{A^{2}} \\
\vdots & =\vdots
\end{aligned}
$$

Here, the superscript of the electric potential $\phi$ denotes the iteration number. For a large aspect ratio $A$, the $\phi_{n}$ will converge to the solution of Eq. 12 within a few iterations.

During each iteration, we need to solve a two-dimensional Poisson equation with the updated source term. The periodic boundary condition for the potential along the $\theta$ direction suggests the use of a complex exponential eigenfunction in that direction. A Bessel function is an appropriate eigenfunction in the radial direction for a round conducting pipe. Hence, we can approximate the potential $\phi$ and source term $\rho$ as follows,

$$
\begin{aligned}
& \rho_{n}(r, \theta)=\sum_{m=-N_{m} / 2}^{N_{m} / 2-1} \sum_{l=1}^{N_{l}} \rho_{n}^{l m} J_{m}\left(\gamma_{l m} r\right) \exp (-i m \theta) \\
& \phi_{n}(r, \theta)=\sum_{m=-N_{m} / 2}^{N_{m} / 2-1} \sum_{l=1}^{N_{l}} \phi_{n}^{l m} J_{m}\left(\gamma_{l m} r\right) \exp (-i m \theta),
\end{aligned}
$$

where $\gamma_{l m}$ is a solution of

$$
J_{m}\left(\gamma_{l m}\right)=0
$$

The $\rho_{n}^{l m}$ and $\phi_{n}^{l m}$ are determined from

$$
\rho_{n}^{l m}=\frac{1}{\pi J_{m}^{\prime 2}\left(\gamma_{l m}\right)} \int_{0}^{2 \pi} \int_{0}^{1} \rho_{n}(r, \theta) \exp (i m \theta) r J_{m}\left(\gamma_{l m} r\right) d r d \theta
$$




$$
\phi_{n}^{l m}=\frac{1}{\pi J_{m}^{\prime 2}\left(\gamma_{l m}\right)} \int_{0}^{2 \pi} \int_{0}^{1} \phi_{n}(r, \theta) \exp (i m \theta) r J_{m}\left(\gamma_{l m} r\right) d r d \theta
$$

For the first iteration, multiplying Eq. 13 by $\exp (i m \theta) r J_{m}\left(\gamma_{l m} r\right)$ and integrating from 0 to $2 \pi$ and 0 to 1 , we obtain

$$
\phi_{n}^{l m}=\rho_{n}^{l m} / \gamma_{l m}^{2}
$$

A similar expansion can be applied to the next iterations except that the source terms in the two-dimensional Poisson equation include the contributions from the charge density functions and from the electric potentials calculated in the last iteration.

For the charged beam in a bent conducting pipe, the electrical potential will have the same boundary conditions as that in the straight pipe except that in this case the longitudinal coordinate is related to the arc length along the bend. A schematic plot of the charged particles inside a bent conducting pipe is shown in Fig. 1. The bending radius $R$ is large compared with the transverse pipe radius $a$. The bunch length of the charged particles is also significantly larger than the pipe radius. Using Frenet-Serret coordinates, the dimensionless 3D Poisson equation can be rewritten as

$$
\nabla_{\perp}^{2} \phi+\frac{1}{R} \frac{1}{1+\frac{r \cos (\theta)}{R}}\left(\cos (\theta) \frac{\partial \phi}{\partial r}-\frac{\sin (\theta)}{r} \frac{\partial \phi}{\partial r}\right)+\frac{1}{\left(1+\frac{r \cos (\theta)}{R}\right)^{2}} \frac{\partial^{2} \phi}{\partial z^{2}}=-\rho
$$

Here, $R=R_{0} / a$ is the normalized curvature radius with $R_{0}$ the physical curvature radius. Approximating the electric potential $\phi$ and charge density $\rho$ in the longitudinal direction by the scaled Hermit-Gaussian series, we obtain for each mode $n$ a group of coupled twodimensional partial equations similiar to Eqs 13-15:

$$
\begin{gathered}
\nabla_{\perp}^{2} \phi_{n}+\frac{1}{R} \frac{1}{1+\frac{r \cos (\theta)}{R}}\left(\cos (\theta) \frac{\partial \phi_{n}}{\partial r}-\frac{\sin (\theta)}{r} \frac{\partial \phi_{n}}{\partial r}\right)+ \\
\frac{1}{\left(1+\frac{r \cos (\theta)}{R}\right)^{2}}\left(\frac{1}{4} \phi_{n-2}-\frac{1}{2}(2 n+1) \phi_{n}+(n+2)(n+1) \phi_{n+2}\right) \frac{1}{A^{2}}=-\rho_{n}
\end{gathered}
$$

These equations can be solved using an iterative procedure:

$$
\begin{aligned}
\nabla_{\perp}^{2} \phi_{n}^{1}= & -\rho_{n} \\
\nabla_{\perp}^{2} \phi_{n}^{2}= & -\rho_{n}-\frac{1}{R} \frac{1}{1+\frac{r \cos (\theta)}{R}}\left(\cos (\theta) \frac{\partial \phi_{n}^{1}}{\partial r}-\frac{\sin (\theta)}{r} \frac{\partial \phi_{n}^{1}}{\partial r}\right)+ \\
& \left(\frac{1}{4} \phi_{n-2}^{1}-\frac{1}{2}(2 n+1) \phi_{n}^{1}+(n+2)(n+1) \phi_{n+2}^{1}\right) \frac{1}{\left(1+\frac{r \cos (\theta)}{R}\right)^{2}} \frac{1}{A^{2}} \\
\nabla_{\perp}^{2} \phi_{n}^{3}= & -\rho_{n}-\frac{1}{R} \frac{1}{1+\frac{r \cos (\theta)}{R}}\left(\cos (\theta) \frac{\partial \phi_{n}^{2}}{\partial r}-\frac{\sin (\theta)}{r} \frac{\partial \phi_{n}^{2}}{\partial r}\right)+
\end{aligned}
$$




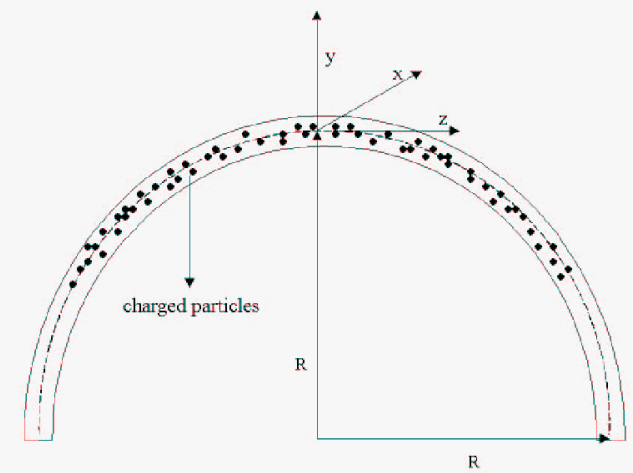

FIG. 1: A schematic plot of the charged particles inside a bent pipe.

$$
\begin{aligned}
& \left(\frac{1}{4} \phi_{n-2}^{2}-\frac{1}{2}(2 n+1) \phi_{n}^{2}+(n+2)(n+1) \phi_{n+2}^{2}\right) \frac{1}{\left(1+\frac{r \cos (\theta)}{R}\right)^{2}} \frac{1}{A^{2}} \\
\vdots= & \vdots
\end{aligned}
$$

Now there are two parameters, $1 / R$ and $1 / A^{2}$, affecting the convergence of the iteration. For most high intensity storage ring accelerators, the physical curvature is of order 100 meters while the transverse pipe radius is about several centimeters. This leads to $R$ in the range of $1000-10000$. For a long bunch which normally occupies a fraction of the ring, the above iteration for each mode will converge quickly.

\section{NUMERICAL TESTS}

The numerical algorithms dicussed in the preceding section are tested using a Gaussian charge distribution in a round conducting pipe. The Gaussian distribution closely approximates the charged particle distribution observed in real accelerator operation. For the convenience of comparison with the Green function solution in a straight conducting pipe, we assume that the beam is axisymmetric. The charge density function is given as

$$
\rho(r, z)=\left\{\begin{aligned}
\exp \left(-\frac{1}{2}\left(\frac{r^{2}}{0.1^{2}}+\frac{z^{2}}{A^{2}}\right)\right) & : \quad r<1 \\
0.0 & : \quad r \geq 1
\end{aligned}\right.
$$


Here, the beam has a normalized transverse rms size 0.1 and a normalized longitudinal rms size $A$. In a straight conducting pipe, the analytical solution for the axisymmetrical Poisson equation using a Green function method is given by

$$
\phi(r, z)=\frac{1}{a \epsilon_{0}} \sum_{n=1}^{n=\infty} \frac{J_{0}\left(\beta_{n} r\right)}{\alpha_{n} J_{1}^{2}\left(\alpha_{n}\right)} \int_{-\infty}^{+\infty} \int_{0}^{\infty} J_{0}\left(\beta_{n} r_{s}\right) \exp \left(-\beta_{n}\left|z-z_{z}\right|\right) \rho\left(r_{s}, z_{s}\right) r_{s} d r_{s} d z_{s}
$$

where $J_{0}\left(\alpha_{n}\right)=0$ and $\beta_{n} a=\alpha_{n}[10]$.

To see the effects of the longtidutinal-to-transverse aspect ratio on the convergence of the coupled two-dimensional Poisson's equations, we have given in Fig. 2 the iteration error as a function of iteration number for aspect ratio $A=2,10,100,1000$ using the charge distribution in Eq. 27. Here the iteration error at step $i$ is defined as $E r_{i}=\sum_{n=0}^{N} \mid \phi_{n}^{i}(r, z)-$ $\phi_{n}^{i-1}(r, z) \mid$ with initial $\phi_{n}^{0}=0.0$, where $\|$ denotes the norm 1 of a matrix. We see that with increase of the aspect ratio, the iteration has converged rapidly. For even a moderate aspect ratio $A=10$, it takes less than 10 iterations to reach an iteration error $10^{-10}$. In this test, the longitudinal mode number for the scaled Hermite-Gaussian series is 10 . The longitudinal mode number required for the solution of the above example depends on the size of aspect ratio $A$. Fig. 3 shows the normalized electric potential as a function of $z$ on the axis from the numerical solution with maximum longitudinal mode number 2,6 , and 10 , for aspect ratio $A=2$ (left) and $A=10$ (right). In this figure, we also show the solution from the analytical Green function calculation for comparison. We see that for small aspect ratio $A=2$, as the Hermite-Gaussian mode number increases from 2 to 10, the numerical solutions gradually approach the Green function solution. For aspect ratio $A=10$, the numerical solutions from all three maximum mode numbers are in excellent agreement with the Green function solution. In Fig. 4, we give a comparison of the numerical solutions and the Green function solutions as a function of $z$ (left) at $r=0,0.25,0.5$, and 0.75 , and as a function of $r$ (right) at $z=0$ and $1.875 A$ with $A=10$ in the above example. We see that the two approaches agree very well at all locations. However, the Green function approach is much slower than the numerical method we proposed here.

The numerical algorithm for a long bunch in the bent conducting pipe has also been tested using the Gaussian charge distribution given in Eq. 27. Figure 5 shows the iteration error as a function of iteration number for the normalized curvature radius $R=2,5,10$, and 100 . The longitudinal-to-transverse aspect ratio is 10 in this case with maximum longitudinal HermiteGaussian mode number 10 . As the curvature radius increases, the iteration converges quickly. 


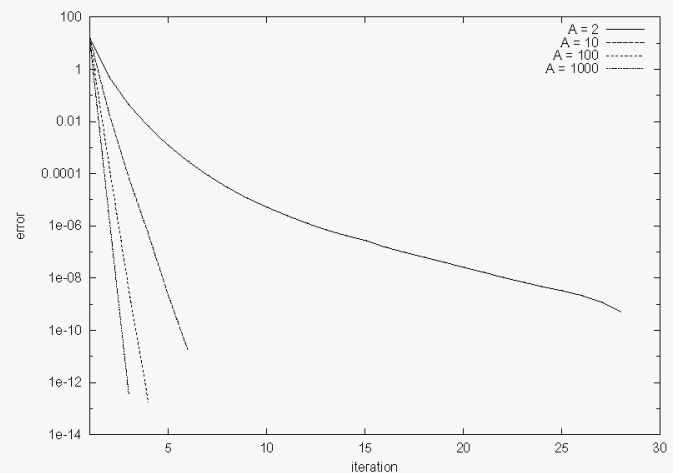

FIG. 2: The norm 1 errors as a function of iteration number for aspect ratio $A=2,10,100,100$.
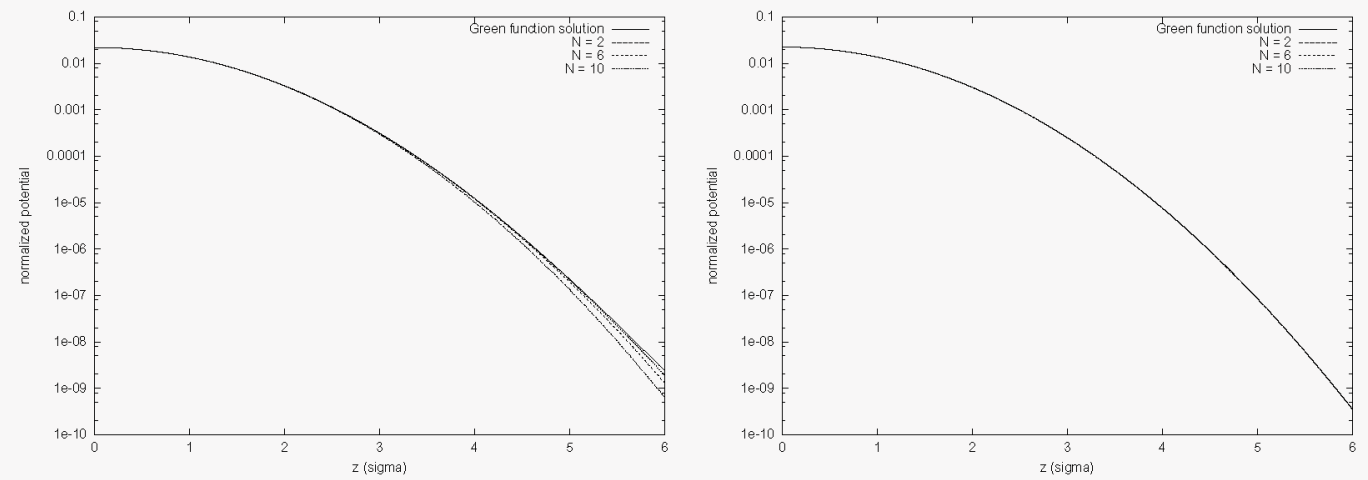

FIG. 3: The normalized electric potential as a function of $z$ on the axis with Hermite-Gaussian mode number 2, 6, and 10 for aspect ratio $A=2$ (left) and 10 (right).

Comparing with Fig. 2, we see that the convergence with increasing curvature radius is not as fast as that with increasing aspect ratio $A$. This is because that the contributions from the curvature term in the preceding iteration scale as $1 / R$ for a fixed aspect ratio in the bent pipe while contributions from the coupling term scale as $1 / A^{2}$ in the straight conducting pipe. Figure 6 gives the numerical solutions of the normalized electric potential as a function of $z$ (left) at $r=0.5$ and as a function of $r$ (right) on the axis for $R=2,5,10,100$ in a bent conducting pipe together with the solutions for a straight pipe. We see that as the curvature radius increases, the numerial solutions in the bent conducting pipe gradually approach those in the straight pipe. For $R=100$ in a bent pipe, there is little difference between the two solutions. This suggests that for large curvature radius as used in most store ring accelerators, the effect of the curvature is very weak in the solution of the $3 \mathrm{D}$ Poisson equation. 

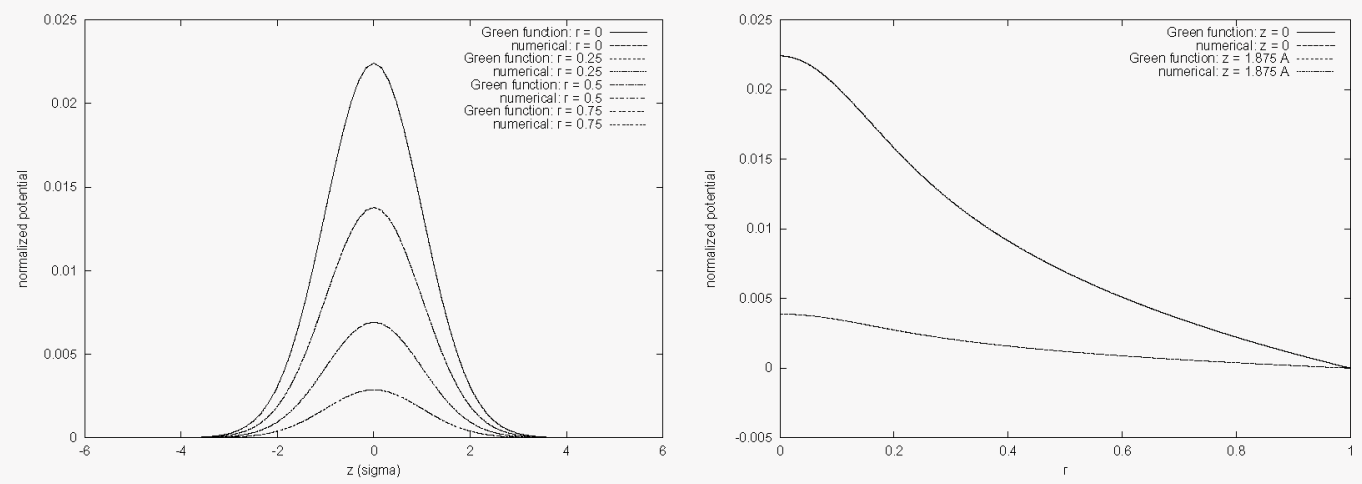

FIG. 4: The normalized electric potential as a function of $z$ at $r=0,0.25,0.5$, and 0.75 , and as a function of $r$ (right) at $z=0$ and $1.875 A$ with $A=10$ together with the Green function solution.

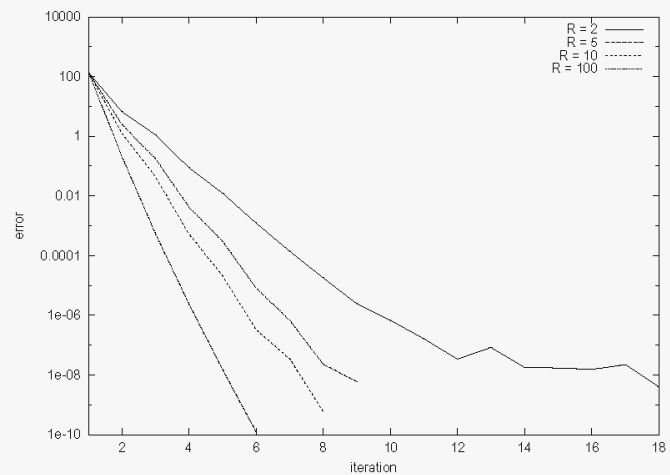

FIG. 5: The norm 1 errors as a function of iteration number for the normalized curvature radius $R=2,5,10$, and 100 .
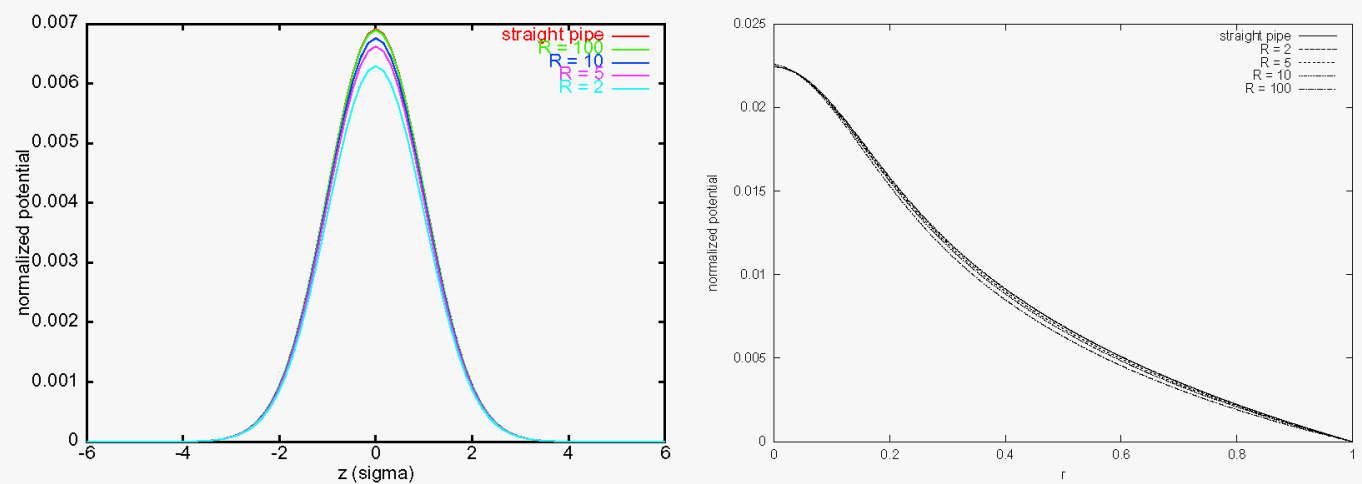

FIG. 6: The numerical solutions of the normalized electric potential as a function of $z$ (left) at $r=0.5$ and as a function of $r$ (right) on axis for $R=2,5,10,100$ in a bent conducting pipe together with the solutions from a straight pipe. 


\section{CONCLUSIONS}

In this paper, we presented a three-dimensional Poisson solver for the electrostatic potential of a charged beam in a straight and a bent conducting pipe with open-end boundary conditions. Using a Hermite-Gaussian series to represent the longitudinal dependence of the electric potential and charge density distribution, the original three-dimensional Poisson equation is reduced to a group of coupled two-dimensional PDEs. With large longitudinalto-transverse aspect ratio, the coupling is weak. The resulting two-dimensional PDEs can be solved independently using an iterative approach, which converges rapidly (within a couple of iterations). The same iterative procedure is also used to solve the three-dimensional Poisson equation in a bent conducting pipe. The rate of convergence in this case depends on both the bunch aspect ratio and the normalized curvature radius. For the physical applications in most accelerators, both the aspect ratio and the normalized curvature radius are large enough to guarantee fast convergence of the iteration. In the solution of the two-dimensional Poisson equation, we have used a Bessel function approximation and a Fourier function approximation for the round conducting cross section. In general, the same Hermite-Gaussian approximation followed by an iterative procedure can also be applied to solving the three-dimensional Poisson equation subject to other type of transverse boundary conditions.

\section{ACKNOWLEDGMENTS}

This research used resources of the National Energy Research Scientific Computing Cen-

ter, which is supported by the Office of Science of the U.S. Department of Energy (US DOE/SC) under Contract No. DE-AC03-76SF00098. This work was performed under the auspices of a Scientific Discovery through Advanced Computing project, "Advanced Computing for 21st Century Accelerator Science and Technology, which is supported by the US DOE/SC Office of High Energy and Nuclear Physics and the Office of Advanced Scientific Computing Research.

\section{REFERENCES}

[1] A. Friedman, D. P. Grote and I. Haber, Phys. Fluids B 4, 2203 (1992). 
[2] T. Takeda, J. H. Billen, "Recent developments of the accelerator design code PARMILA," Proceedings of the XIX International Linac Conference, Chicago, IL, August 1998 , pp. 156-158.

[3] J. Qiang, R. D. Ryne, S. Habib, V. Decyk, J. Comput. Phys. 163, 434 (2000).

[4] D. B. Haidvogel and T. Zang, J. Comput. Phys. 30, 167 (1979).

[5] S. Ohring, J. Comput. Phys. 50, 307 (1983).

[6] H. Dang-Vu and C. Delcarte, J. Comput. Phys. 104, 211 (1993).

[7] E. Braverman, M. Israeli, A. Averbuch, and L. Vozovoi, J. Comput. Phys. 144, (1998).

[8] L. Plagne and J. Berthou, J. Comput. Phys. 157, 419 (2000).

[9] J. Qiang and R. D. Ryne, Computer Physics Communications 138, 18 (2001).

[10] C. K. Allen, N. Brown, and M. Reiser, Particle Accel. 45, 149 (1994). 